\title{
Label-free optical biosensor for direct complex DNA detection using Vitis vinifera L.
}

\author{
Luis Moreira $^{\mathrm{a}}$, Helena M.R. Gonçalves ${ }^{\mathrm{a}, \mathrm{b}}$, Leonor Pereira ${ }^{\mathrm{a}, \mathrm{b}}$, Cláudia Castro $^{\mathrm{a}}$, Pedro Jorge ${ }^{\mathrm{c}}$, \\ Carlos Gouveia ${ }^{c}$, José R. Fernandes ${ }^{a, c}$, Paula Martins-Lopes ${ }^{a, b, *}$ \\ a University of Trás-os-Montes and Alto Douro, P.O. Box 1013, 5000-911 Vila Real, Portugal \\ ${ }^{\mathrm{b}}$ University of Lisboa, Faculty of Sciences, BioISI-Biosystems E Integrative Sciences Institute, Campo Grande, Lisboa, Portugal \\ ' INESC TEC, Rua do Campo Alegre n. 687, 4169-007 Porto, Portugal
}

\section{A R T I C L E I N F O}

\section{Article history:}

Received 28 December 2015

Received in revised form 12 April 2016

Accepted 17 April 2016

Available online 23 April 2016

\section{Keywords:}

Label-free biosensor

DNA detection

Quantification

Vitis vinifera $\mathrm{L}$

\begin{abstract}
A B S T R A C T
The ability to detect and quantify small amounts of DNA in biological complex samples is a hot research area. Up until recently most of the work performed in this area used label-dependent protocols that increases its complexity and overall costs. The aim the work was to develop a label-free technology suitable for DNA detection and quantification using real complex DNA samples. The applicability of this system was tested using synthetic ssDNA targets that guaranteed the systems specificity, in the sense that only complementary sequences hybridized with the probe. When using real samples extracted from Vitis vinifera $\mathrm{L}$. the system was able to successfully detect and quantify the DNA present without any of the time consuming and costly amplification steps. The detection and quantification limits of the proposed system were $60 \pm 20 \mathrm{nM}$ and $201 \pm 20 \mathrm{nM}$, respectively for Target 1 concentrations between 31 and $350 \mathrm{nM}$. This method can easily be applied to other species and purposes, allowing the direct detection of DNA in a label-free environment with high accuracy and specificity.
\end{abstract}

(c) 2016 Elsevier B.V. All rights reserved.

\section{Introduction}

Ever since the discovery of the DNA base-pair coupling structure, the number of publications where this complex biomolecule is used as a sensing device has grown exponentially. One area that has taken a major interest in the DNA base-paring structure is the development of biosensors [1,2]. The use of DNA as a structural base for biosensing development has numerous advantages, such as: (1) the thermostability of the molecule when compared to other biomolecules, i.e. proteins; (2) highly conserved in the sense that replication has a very low mutation rate, allowing an accurate fingerprinting (3) the presence in all the cells, thereby potentially enabling identical information regardless of the tissue origin and the transformation process that they were submitted $[3,4]$.

A DNA-based biosensor can be of the outmost importance in areas such as, food traceability and authentication, clinical diagnostics, gene therapy, biomedical studies, among others [5-8].

Over the past few years there has been some efforts in order to develop biotechnologies that will improve both sensitivity and

\footnotetext{
* Corresponding author at: University of Trás-os-Montes and Alto Douro, P.O. Box 1013, 5000-911 Vila Real, Portugal.

E-mail addresses: plopes@utad.pt, pfmlopes@hotmail.com (P. Martins-Lopes).
}

selectivity when considering gene analysis [8-10]. Usually these analysis require DNA labelling with a fluorophore. In some particular cases where signal amplification is the most critical issue, such as, ultratrace gene analysis, the DNA hybridization is followed using a fluorescent dye $[1,11]$. However fluorophores have numerous disadvantages that have reflection's in the sensitivity of the method, namely low photostability, photobleaching, low signal amplification, particularly in biological tissue where self-fluorescence is a major issue, among others [12]. Moreover, the DNA probe can only be labelled with one or a few fluorophores, which results in a weak signal, particularly when the target concentration is low. This limits not only affect the method's sensitivity but also the detection and quantification limits.

In order to overcome these issues, some work has been developed in terms of nanoparticles to support the DNA or a fluorescence label. In fact, and even though some interesting values have been reported $[1,6,13,14]$ for the detection limit, these alternatives still require DNA labelling.

Other DNA-based technologies rely on amplification procedures, based on Polimerase Chain Reaction (PCR) previous to detection, such as real time PCR that additionally requires an amplification in order to detect the target and quantify it, with some success limitation when PCR-inhibitors are present in the sample 
Table 1

Sequences of the oligonucleotides used throughout this work

\begin{tabular}{|c|c|}
\hline Oligonucleotides & Sequence \\
\hline ssDNA - Probe & 5'-C6-Aminolink GGTGAAATGGGCACCGAACACACGC-3' \\
\hline Target $_{1}$ (Complementary) & 5'-GCGTGTGTTCGGTGCCCATTTCACC-3' \\
\hline Target $_{2}$ (Non-Complementary) & 5'-AAAAAAAAAAAAAAACCATTTCACC-3' \\
\hline Target $_{3}$ (One Base Mismatch) & 5'-GCATGTGTTCGGTGCCCATTTCACC-3' \\
\hline Target $_{4}$ (Six Bases Mismatch) & 5'-GCATGTGTTTTTTGCCCATTTCACC-3' \\
\hline Target 5 (Complementary with a tail of 24 bases in $5^{\prime}$ ) & 5' - TCTCTCTCTCCTCTCAGCAAGGAAGCGTGTGTTCGGTGCCCATTTCACC - 3' \\
\hline
\end{tabular}

[15]. Alternative to these methods is the use of fiber optic SPR for DNA hybridization detection [16] however, these options require expensive equipment and specialized personnel.

In this work a simple, low cost method for label-free DNA detection and quantification was developed, producing competitive results. This method is based on the ssDNA (DNA-probe) immobilization in the lateral surface of an optical fiber long period grating (LPG) and subsequent hybridization. The DNA is not dyelabelled and the hybridization is followed in situ by differences induced on the optical fiber surrounding media refractive index. More, the process does not require enzymatic reactions. This system was successfully applied for the detection of synthetic ssDNA targets (complementary, non-complementary and partiallycomplementary), as well as, genomic DNA previously extracted from Vitis vinifera $\mathrm{L}$.

\section{Material and methods}

\subsection{Materials}

All oligonucleotides used in this work were purchased from Frilabo. The stock solutions were prepared with ultrapure water and stored at $-20^{\circ} \mathrm{C}$. Each solution contained $100 \mu \mathrm{M}$ of each oligonucleotides. The oligonucleotides sequences are presented in Table 1 and was based on the specific primer of $V$. vinifera designed to detect Single Sequence Repeats [17].

For each experiment a suitable amount of the stock solution was diluted in saline phosphate buffer (PBS: $10 \mathrm{mM}$ sodium phosphate; $120 \mathrm{mM} \mathrm{NaCl} ; 2.7 \mathrm{mM} \mathrm{KCl} ; \mathrm{pH} 7.4$ ) in order to obtain the following concentrations: $0.50 ; 0.25 ; 0.125 ; 0.0625 ; 0.03125 ; 0.015625$ and $0.007812 \mu \mathrm{M}$. All other chemicals were used without further dilutions.

The cleaning solution used before each experiment was composed by Ethanol $70 \%(\mathrm{v} / \mathrm{v})$ and $1 \%$ Hydrochloric acid $(\mathrm{v} / \mathrm{v})$ in a $(1: 1)$ ratio. Additionally the restringing solution was a mixture of PBS with $0.1 \times$ Saline-Sodium Citrate (SSC) and 0.1\% Sodium dodecyl sulphate (SDS) in a (1:1) ratio. After each cycle the LPG was cleaned using a diluted solution of Nitric Acid $\left(\mathrm{HNO}_{3} 1: 3\right)$.

\subsection{Instrumentation}

The detection system is based in a fiber Long Period Grating (LPG) sensor. This sensor is an optical wavelength band-loss filter where the central wavelength of the band depends of the LPG fabrication parameters, temperature, applied axial mechanic tension and fiber surrounding media refractive index. It is also known that the double strand DNA has, in solution, a slightly higher refractive index than the single strand form. The detection principle of this particular LPG sensor is based on the use of a single DNA strand (Probe) attached to the fiber's lateral surface. When the probe hybridizes with its complementary strand, it becomes a double strand DNA increasing the fiberís surrounding refractive index media that, in turn, will have an effect on the LPG transmission spectra that is the data measured.

For that purpose the LPG sensor was placed inside of a wet flow cell that will provide the means to maintain a constant mechanic tension applied to the fiber and, simultaneously allows the insertion and removal of wet solutions in the sensor surrounding zone which has been detailed in Gonçalves et al. [18]. As consequence, the wavelength variations accounted can only be due to the interaction of the solution with the LPG surface. The chamber capacity is of $750 \mu \mathrm{L}$ and the same volume of each solution was injected into the chamber in order to maintain the volume constant. The data acquisition was performed using a fiber optic interrogation unit manufactured by Fibersensing ${ }^{\circledR}$, model BraggMeter FS2200SA, with two channels modified to allow the measuring of the transmission spectra in the spectral region between 1500 and $1600 \mathrm{~nm}$.

The sensor apparatus was maintained at constant temperature by inserting it in a muffle (Termarks, model B 8023). The sensor temperature was measured by a type-K thermocouple positioned in contact with the sensor chamber and its value were recorded by a temperature logger (Keithley ${ }^{\circledR} 740$ ) controlled by the same computer that was measuring the spectra using a Labview ${ }^{\circledR}$ program creating a temperature list with a temporal tag for each temperature value. In this way, both spectra and temperature measurement where time synchronized and can be co-related.

Upon data acquisition the spectral data is processed to determine the position of the wavelength LPG resonance as described by Gonçalves et al. [18].

\subsection{DNA extraction}

In order to test the applicability of the sensing system into a more complex DNA matrix, a sample of the Vitis vinifera L. DNA was tested. The genomic DNA was extracted from leaf samples using the cetyl trimethylammonium bromide (CTAB) method described by Doyle and Doyle [19]. The extracted DNA was resuspended in $50 \mu \mathrm{L}$ of distilled water and the DNA concentration was determined using a NanoDrop ${ }^{\circledR}$ ND-1000 spectrophotometer. The DNA quality was assessed using a $0.8 \%(\mathrm{w} / \mathrm{v})$ agarose gel stained in $7 \mu \mathrm{g} \mathrm{mL}^{-1}$ ethidium bromide solution.

\subsection{Chemical sandwich sensing system preparation}

The LPGs were inserted in a glass chamber and the strain was fixed. Before performing any tests the LPG surface was cleaned by the passage of ethanol $70 \%(\mathrm{v} / \mathrm{v})$ and hydrochloric acid $1 \%(\mathrm{v} / \mathrm{v})$ solution in a $1: 1$ ratio.

The LPG surface is negatively charged, so is the DNA, as such, it was necessary to use a bilinker. In this work, the bilinker chosen was Poly-L-Lysine (PLL). In each cycle the following sequence was used: water, PLL, Probe, Targets or DNA from $V$. vinifera. All samples used were set at $0.25 \mu \mathrm{M}$. All measurements, with the exception of water added in between the addition of an analyte, were performed over $30 \mathrm{~min}$, at $37^{\circ} \mathrm{C}$. Upon the hybridization the target was removed from the LPG surface by a process called stripping. This was performed at $60^{\circ} \mathrm{C}$ and included the addition of a stripping solution (0.1x Saline-Sodium Citrate and $0.1 \%$ Sodium Dodecyl Sulphate in a (1:1) ratio), ethanol and water. In the end of each cycle all chemicals were removed from the LPG surface, using a diluted solution of $\mathrm{HNO}_{3}(1: 3, \mathrm{v} / \mathrm{v})$ that was added and left in contact with the LPG for $15 \mathrm{~min}$. 


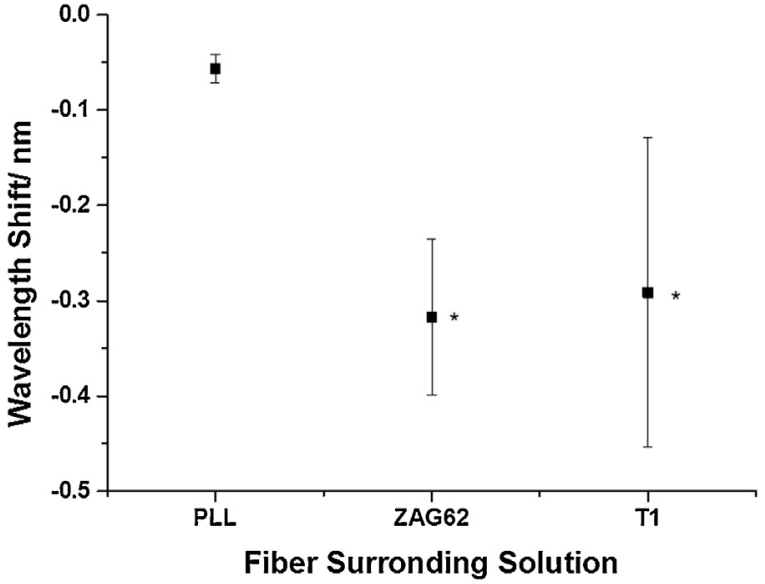

Fig 1. Representation of the signal obtained using a simple ssDNA (Probe), an aminoterminated ssDNA(ZAG62) and Target ${ }_{1} .{ }^{*}$ The values are statistically significant when compared to the Probe Signal for a $\mathrm{p}<0.05$.

\subsection{Chemical sandwich sensing system preparation}

In order to evaluate the statistical significance of the data obtained throughout this work an IBM Software Package Statistical Analysis (SPSS) version 19 was used. The data were analysed in comparison to the Probe and the PLL signal, for the targets and the ZAG62 evaluation, respectively, and were considered statistical different when $\mathrm{p}<0.05$.

\section{Results and discussion}

\subsection{The chemical interaction between the sensing system}

A common problem in immobilized biomolecule methods is the need that even though the DNA-probe is covalently-attached to the fiber it should still interact with the targets and hybridize. This problem was surpassed by the use of a polymer-Poly-LLysine (PLL) [20]. This polymer has a positive charge which makes it suitable for a single sandwich system-fiber (negative charge); PLL (positive charge); DNA-probe (negative charge). Indeed, even though PLL is commonly used as a bilinker for DNA attachment, the chemical interactions between this system are not fully understood. In order to better understand the chemical relationship that lied beneath the sandwich sensing system an experiment was devised. According to authors, such as Zibaii et al. [20], PLL forms a monolayer that links to the optical fiber surface through a hydrogen bridge bound between the $\mathrm{Si}-\mathrm{OH}$ groups of the fiber and the PLL amino group. Additionally these authors suggest that the linking between DNA and PLL also occurs through an amino link. However what remains to be explained is if the absence of the amino-terminated groups in the DNA is determinant for the hybridization to occur. We wanted to test this hypothesis and used amino-ssDNA and DNA without an amino group. The results presented in Fig. 1, show that their statistical difference between the PLL signal and the amino free DNA (ZAG62), as such, the ZAG62 can effectively interact with the immobilized PLL even though it does not have an amino group. However, when comparing these signals with Target ${ }_{1}$ it is possible to say that there is no statistical confirmation for hybridization. This behaviour can be due to an orientation effect that the amino group confers to the ssDNA. Indeed the interaction between the PLL and the ZAG62 or Probe, can be due to hydrogen bounding between the DNA residues and the polymer, but when there is an amino group in the ssDNA extremity (Probe), the interaction between PLL and the Probe is more significant due to the stability of the $\mathrm{N}-\mathrm{N}$ chemical bounding. Additionally, when

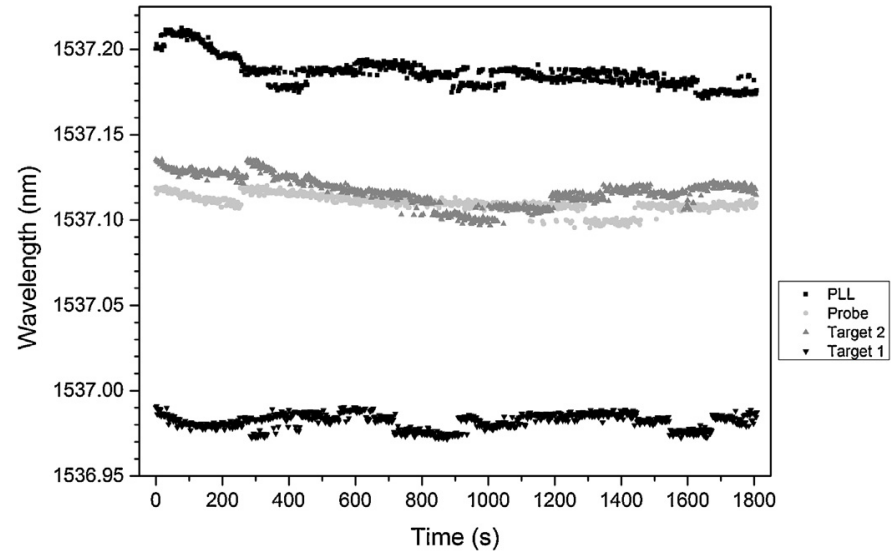

Fig. 2. Representation of the wavelength evolution with time registered for 30 min within each step PLL Deposition, Probe immobilization, Target 2 (noncomplementary) and Target ${ }_{1}$ (hybridization).

the interaction between the polymer and the DNA is due to this $\mathrm{N}-\mathrm{N}$ bounding the position for this interaction favours the subsequent hybridization. On the other hand, when the ssDNA and the PLL is not specifically oriented this interaction can place the sSDNA (ZAG62) in such a way that the hybridization cannot be performed. This position is so variable that it is possible to see a hybridization in one cycle and not see it in the next, which can be responsible to the high standard deviation found for Target ${ }_{1}$ in these particular experiments.

\subsection{Stability and reproducibility of the sensing system}

The stability of the sensor is an important issue. In order to define the time requested to obtain a stable signal a study concerning a time frame of $1800 \mathrm{~s}$ was undertaken using PLL, Probe, Target 2 and Target 1 . The wavelength signal was recorded during $30 \mathrm{~min}$ (Fig. 2) and stabilization of the reaction on the fiber within each analyte was obtained after $20 \mathrm{~min}$. All the further readings were done taking into consideration this time frame.

Another issue that needed to be addressed was the low reproducibility that is often reported when using fibers with long period gratings (LPGs), depending on the thickness of the interaction region and the penetration depth of the evanescent [21]. Indeed when using different LPGs it is necessary to perform a calibration that can be slow due to its singular nature. In order to overcome this problem a new strategy was developed, where the LPG was put in contact with a diluted solution of nitric acid. This allowed the use of the same LPG for more than 20 complete assays over more than 5 months.

In order to evaluate the method sensitivity and selectivity five different strands of DNA were tested. Target ${ }_{1}$, Target ${ }_{2}$, Target ${ }_{3}$, Target ${ }_{4}$ and Target ${ }_{5}$ (Table 1 ). Target ${ }_{1}$ is complementary to the DNA-probe, Target ${ }_{2}$ is non-complementary, Target ${ }_{3}$ has a singlemismatch close to the $5^{\prime}$ end, Target ${ }_{4}$ has 6 mismatches and Target 5 is complementary, but it has a tail of 24 extra-bases in the $5^{\prime}$ end. The hybridization process was followed by monitoring the change in the effective refractive index induced by the events.

In order to ascertain the method reproducibility, sensitivity, detection and quantification limits several amounts of Target 1 were evaluated by the same fiber sensor. This allowed the definition of the calibration curve presented as Supplementary Fig. S1 in the online version at DOI: $10.1016 / j . s n b .2016 .04 .105$ with a $\mathrm{R}^{2}$ of 0.98 The obtained results allowed us to determine the method's Detection Limit: $60 \pm 20 \mathrm{nM}$ and Quantification Limit: $201 \pm 20 \mathrm{nM}$. These limits are quite low for a label-free technology. Indeed, even though detection limits in the hundreds of fentomolars have been reported 


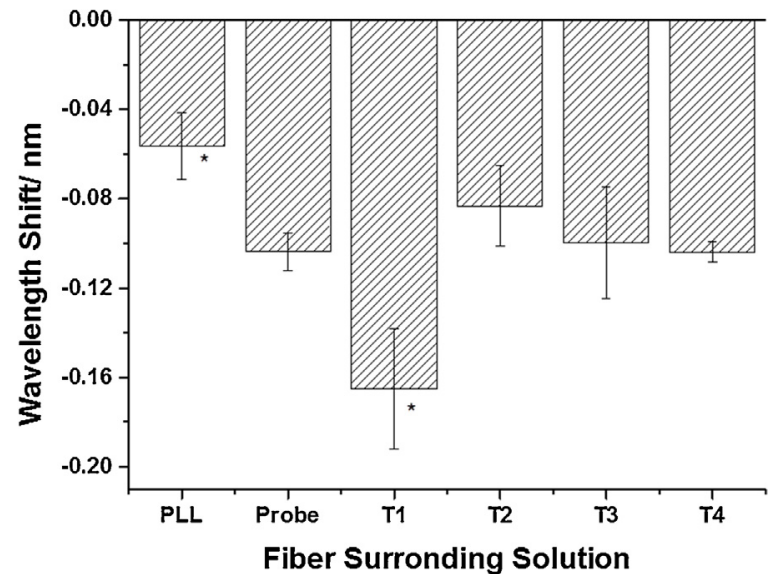

Fig. 3. Representation of the sensor response in the presence of Poly-L-Lysine (PLL),

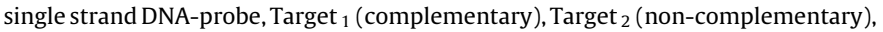
Target $_{3}$ (single mismatch) and Target ${ }_{4}$ (six mismatches), for three independent measurements. *The values are statistically significant when compared to the Probe Signal for a $\mathrm{p}<0.05$.

[22-24]. All these methods required DNA labelling, increasing costs and requiring additional labelling steps. Moreover, our method also allows the quantification of the DNA in a small sample, without requiring a previous amplification process as required in Real-Time PCR assays [15] and in other biosensor based methods [22].

\subsection{Analytical performance of the sensing system}

In order to evaluate the applicability of the sensing system several targets were used. Each target had a peculiarity so it could be possible to ascertain if the developed system was able to detect single mismatch, if the mismatch position was relevant and if complementary sequence could be compromised by having a tail of mismatches in a $5^{\prime}$ end.

The results showed in Fig. 3 clearly demonstrate that hybridization only occurs for Target ${ }_{1}$, using a concentration of $0.25 \mu \mathrm{M}$. This result is quite interesting since it allows to conclude that our system can successfully discriminate between total-complementarity and a single mismatch. Target ${ }_{4}$ had 6 single mismatches along the chain that could easily prevent hybridization. This result clearly shows that the method is very selective, which is reinforced by the result obtained for Target 3 which has only one mismatch. This is interesting once it can be considered as a new biosensor method for Single Nucleotide Polymorphism (SNP) detection [22,25], which can be applied in a wide range of research areas, from diagnose, forensic, genotyping, among others. Another advantage is that it does not require the development of complicated probes, e.g. Peptide Nucleic Acid (PNA), Locked Nucleic Acid (LNA), nor the use of signal amplification post hybridization process, e.g. Surface Ligation Reaction [22].

To help establish the use of this method in a real sample, a suitable amount of DNA was extracted from $V$. vinifera. This is a widely cultivated fruit crop with a harvested area above seven million hectares and more than 60 million tons of grapes produced per year. It is a diploid species with nineteen chromosomes, with a genome size of around $500 \mathrm{Mbp}$. Additionally, Target 5 was used, and although its sequence is complementary it has a tail of 24 bases on the $5^{\prime}$ end, simulating interference of non-complementary sequences surrounding the complementary sequence, present in real DNA samples. Nevertheless, Target 5 only had a tail in one of the sequence end. As it can be seen by the analysis of Fig. 4, there is a statistical significant difference between the signal obtained for the ssDNA and Target ${ }_{5}$. This result suggests that the sensing system

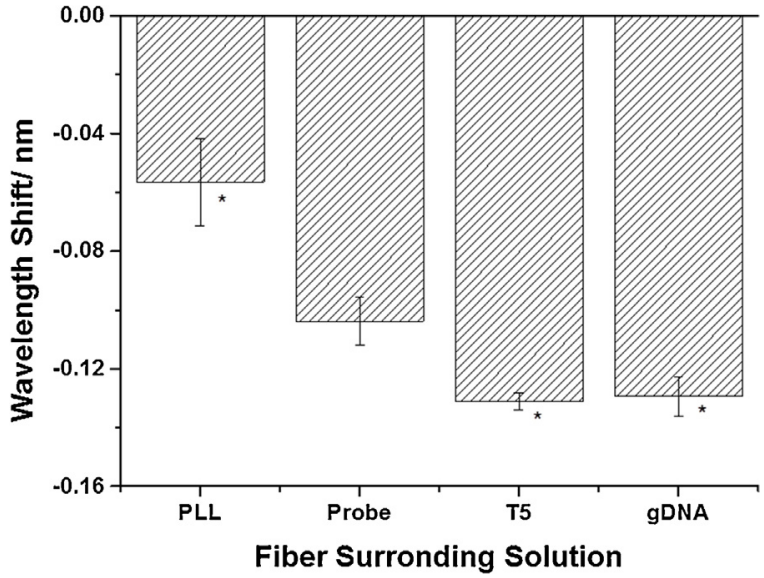

Fig. 4. Representation of the sensor response) in the presence of Poly-L-Lysine (PLL), single strand DNA (Probe), Target ${ }_{5}$ (complementary with a 24 bases tail in $5^{\prime}$-end) and Genomic DNA (DNA extracted from Vitis vinifera $\mathrm{L}$.), for three independent measurements. ${ }^{*}$ The values are statistically significant when compared to the Probe Signal for a $\mathrm{p}<0.05$.

identified this target as complementary to the ssDNA immobilized in the fiber.

The procedure applied to analyze the genomic DNA extracted from $V$. vinifera was very similar to the one used for the previous targets. However, since the DNA was still double stranded a denaturation process was applied. This was accomplished by a simple heating of the DNA sample. In order to evaluate the competitive mechanism between the immobilized probe and the two singlestranded DNA chains from the $V$. vinifera DNA sample, they were both put into contact with the LPG surface. The response observed for this sample (Fig. 4) clearly demonstrates that despite the competitive existent due to the presence of complementary sequence, the designed system can indeed detect the hybridization process.

When comparing the signal differences between the probe, Target ${ }_{1}$ and the real DNA sample it is possible to ascertain the impact that the competitive mechanism has on the system. The hybridization of Target ${ }_{1}$ results in a signal increase of approximately $59 \%$ on the other hand when there is a competitive mechanism between the immobilized probe and the two complementary chains of the real sample, the hybridization is followed by a $20 \%$ signal increase. As such, it is possible to say that our system is able to successfully detect the DNA in a more complex matrix (real sample). Moreover, even though we are dealing with a real sample, it is possible to see that the results are quite reproducible-the highest standard deviation in three consecutive measurements is $2 \%$.

As all the experiments were conducted using the same molarity, $0.25 \mathrm{M}$, the number of estimated copies between the synthetic targets and the real sample are significantly different, being $5.330 \mathrm{E}^{+16}$ for Target ${ }_{1}$, and $2.687 \mathrm{E}^{+6}$ for the real sample. This emphasis the sensibility of the method when using direct real DNA samples, and the fact that no previous amplification step was required, increasing the target number in the solution as is required for some actually used DNA-based biosensors [22]. This is an interesting feature as some DNA samples present PCR-inhibitors [15], limiting their use for genotyping procedures.

\section{Conclusions}

The optical DNA-based biosensor tested proved to be highly specific when synthetic samples were used, detecting a unique SNP difference. The amazing outcome was the fact that this biosensor was successfully applied in a complex matrix, $V$. vinifera genomic DNA, even when the sequence copy number is considerably lower than the synthetic target used and with the presence of 
competitive mechanisms. This system makes it possible to detect and quantify DNA in real samples, using standard optical telecommunication technology, high with specificity, since the method allows the detection at a SNP level, which is why we expect that it would be quite useful in areas where the DNA detection/quantification is the main goal.

\section{Acknowledgements}

This research was supported by the Portuguese Foundation for Science and Technology (FCT) in the project Biosensor Development for Wine Traceability in the Douro Region-WineBiocode PTDC/AGR-ALI/117341/2010-FCOMP-01-0124-FEDER-019439, Enoexcel- NORTE-07-0124-FEDER-000032, funded by national means through ON.2 and co-funded by the European Fund for Regional Development (FEDER) through COMPETE-Operational Program for Competitiveness Factors (POFC) and a PhD grant (SFRH/BD/44781/2008).

\section{References}

[1] X.H. Fang, W. Tan, Imaging single fluorescent molecules at the interface of an optical fiber probe by evanescent wave excitation, Anal. Chem. 71 (1999) 3101-3105.

[2] S. Weiss, Fluorescence spectroscopy of single biomolecules, Science 283 (1999) 1676-1683.

[3] A.K. Lockley, R.G. Bardsley, DNA-based methods for food authentication, Trends Food Sci. Tech. 11 (2000) 67-77.

[4] X. Zhao, R. Tapec-Dytioco, W. Tan, Ultrasensitive DNA detection using highly fluorescent bioconjugated nanoparticles, J. Am. Chem. Soc. 125 (2003) $11474-11475$.

[5] L.M. Reid, C.P. O’Donnell, G. Downey, Recent technological advances for the determination of food authenticity, Trends Food Sci. Tech. 17 (2006) 344-353.

[6] X. Sun, M. Jia, L. Guan, J. Ji, Y. Zhang, L. Tang, Z. Li, Multilayer graphene-gold nanocomposite modified stem-loop DNA biosensor for peanut allergen-Ara h1 detection, Food Chem. 172 (2015) 335-342.

[7] A. Uygun, DNA hybridization electrochemical biosensor using a functionalized polythiophene, Talanta 79 (2009) 194-198.

[8] J. Wang, D. Xu, R. Polsky, Magnetically-induced solid-State electrochemical detection of DNA hybridization, J. Am. Chem. Soc. 124 (2002) 4208-4209.

[9] L. He, M.D. Musick, S.R. Nicewarner, F.G. Salinas, S.J. Benkovic, M.J. Natan, C.D. Keating, Colloidal Au-enhanced surface plasmon resonance for ultrasensitive detection of DNA hybridization, J. Am. Chem. Soc. 122 (2000) 9071-9077.

[10] G.M. Makrigiorgos, S. Chakrabarti, Y.Z. Zhang, M. Kaur, B.D. Price, A PCR-based amplification method retaining the quantitative difference between two complex genomes, Nat. Biotechnol. 20 (2002) 936-939.

[11] M. Safdar, Y. Junejo, Development and validation of fast duplex real-time PCR assays based on SYBER Green florescence for detection of bovine and poultry origins in feedstuffs, Food Chem. 173 (2015) 660-664.

[12] S. Uno, D. Tiwari, M. Kamiya, Y. Arai, T. Nagai, T. Urano, A guide to use photocontrollable fluorescent proteins and synthetic smart fluorophores for nanoscopy, Microscopy 64 (4) (2015) 263-277.

[13] I.L. Medintz, H.T. Uyeda, E.R. Goldman, H. Mattoussi, Quantum dot bioconjugates for imaging, labelling and sensing, Nat. Mat. 4 (2005) 435-446.

[14] X. Zuo, F. Xia, Y. Xiao, K.W. Plaxco, Sensitive and selective amplified fluorescence DNA detection based on exonuclease III-aided target recycling, J. Am. Chem. Soc. 132 (2010) 1816-1818.

[15] P. Martins-Lopes, S. Gomes, L. Pereira, H. Guedes-Pinto, Molecular markers for food traceability, Food Technol. Biotech. 51 (2) (2013) 198-207.

[16] J.D. Suter, I.M. White, H. Zhua, H. Shi, C.W. Caldwell, X. Fan, Label-free quantitative DNA detection using the liquid core optical ring resonator, Biosens. Bioelectron. 23 (2008) 1003-1009.

[17] K.M. Sefc, F. Regner, E. Turetschek, J. Glössl, H. Steinkellner, Identification of microsatellite sequences in Vitis riparia and their applicability for genotyping of different Vitis species, Genome 42 (1999) 367-373.

[18] H.M.R. Gonçalves, L. Moreira, L. Pereira, P. Jorge, C. Gouveia, P. Martins-Lopes, J.R. Fernandes, Biosensor for label-free DNA quantification based on functionalized LPGs, Biosens. Bioelectron. (2015), http://dx.doi.org/10.1016/j. bios.2015.10.001 (Accepted).

[19] J.J. Doyle, J.L. Doyle, A rapid total DNA preparation procedure for fresh plant tissue, Focus 12 (1990) 13-15

[20] M.I. Zibaii, H. Latifi, E. Ghanati, M. Gholami, S.M. Hosseini, Label free detection of DNA hybridization by refractive index tapered fiber biossensor, Proc. SPIE 7715 (2010), 77151Z1-77151Z9.

[21] F. Baldini, M. Brenci, F. Chiavaioli, A. Giannetti, C. Trono, Optical fibre gratings as tools for chemical and biochemical sensing, Anal. Bioanal. Chem. 402 (2012) 109-116.
[22] K. Chang, S. Deng, M. Chen, Novel biosensing methodologies for improving the detection of single nucleotide polymorphism, Biosens. Bioelectron. 66 (2015) 297-307.

[23] H. Fan, Y. Xu, Z. Chang, R. Xing, Q.J. Wang, P.G. He, Y.Z. Fang, A non-immobilizing electrochemical DNA sensing strategy with homogenous hybridization based on the host-guest recognition technique, Biosens. Bioelectron. 26 (2011) 2655-2659.

[24] A.H. Wu, J.J. Sun, R.J. Zheng, H.H. Yang, G.N. Chen, A reagentless DNA biosensor based on cathodic electrochemiluminescence at a C/CxO1-x electrode, Talanta 81 (2010) 934-940.

[25] W. Zhang, T. Yang, K. Jiao, Ultrasensitive indicator-free and enhanced self-signal nanohybrid DNA sensing platform based on electrochemically grown poly-xanthurenic acid/ $\mathrm{Fe}_{2} \mathrm{O}_{3}$ membranes, Biosens. Bioelectron. 31 (2012) 182-189.

\section{Biographies}

Luis Moreira graduated with a degree in Genetics and Biotechnology and M.Sc degree in Food Quality and Biotechnology from the University of Trás-os-Montes and Alto Douro, Portugal, in 2011 and 2013, respectively. From 2013-2015 worked in the Center of Genetics and Biotechnology, Vila Real, Portugal. He has 2 publications in SCI journals and 10 abstracts in national and international meetings.

Helena Gonçalves graduated in Chemistry, received her M.Sc. degree in Chemistry, at the University of Porto, Portugal in 2007 and 2008, respectively. In 2009 attended a post-graduation in Medical and Legal Science and in 2014 concluded her Ph.D. in Chemistry from Porto University, Portugal. She has been involved in 6 research projects. From her research work, Helena is author and co-author of 13 full-papers published SCI journals, 1 patent and several abstracts in national and international meetings.

Leonor Pereira graduated with a degree in Biotechnology Engineering at the Polytechnic Institute of Bragança, Portugal, in 2002. She obtained her M.Sc. degree in Molecular Genetics from the University of Minho, Braga, Portugal in 2006. In 2015 she finished her PhD from the University of Trás-os-Montes and Alto Douro, Portugal. From 2008-2015 she worked in the Centre of Genetics and Biotechnology, Vila Real, Portugal. She participated in 5 projects. She has 7 publications in SCI journals, 3 patents, 1 abstract published in SCI journals, 1 full-paper published in peer-reviewed journal, and 18 abstracts published in national and international meetings.

Cláudia Castro, Graduated with a degree in Genetics and Biotechnology and M.Sc. degree in Comparative Molecular Genetics and Technology from the University of Trás-os-Montes and Alto Douro, Portugal, in 2013 and 2015. She has published 5 abstracts in national and international meetings.

Pedro A. S. Jorge graduated in Applied Physics (Optics and Lasers) at the Univ. of Minho (1996), M.Sc. in Optoelectronics and Lasers at the Physics Department of University of Porto (2000); in 2006 concluded his Ph.D. program at University of Porto in collaboration with the Dept. of Physics and Optical Sciences at the Univ. of Charlotte, North Carolina, USA, with work in luminescence based optical fiber systems for biochemical sensing applications using luminescent nanoparticles. Since 1997 Pedro Jorge has been involved in several research and technology transfer projects related to optical fiber sensing technology, developing new sensing configurations and interrogation techniques for optical sensors. Pedro Jorge is a Senior researcher at INESC Porto where he leads the Biochemical Sensors team exploring the potential of optical fiber and integrated optics technologies in industrial, environmental and medical applications coordinating several projects in these areas. From 2015 he is also an Invited Assistant Professor at the Department of Physics and Astrophysics of the Faculty of Sciences of the University of Porto. Pedro Jorge has more than 200 publications in the fields of sensors in national and international conferences and peer reviewed journals, he is author of 3 book chapters and also holds one patent Pedro Jorge is a member of SPIE.

Carlos Gouveia graduated with a degree in electronics and telecommunications and M.Sc. degree in telecommunications and networks from the University of Madeira, Portugal, in 2007 and 2008, respectively. Since 2008 works in the Center of Applied Photonics of INESC TEC, Porto, Portugal. In January 2014, he obtained his Ph.D. degree in electrical engineering from University of Madeira. Currently the is pos-doc researcher at INESC P\&D Brasil, hosted by the Electrical Engineering Department of Federal University of Campina Grande. He has more than 40 publications in the fields of sensors in national and international conferences and peer reviewed journals, is author of 1 book chapters.

J.R.A. Fernandes graduated in Physics at University of Porto (1994), M.Sc. in Optoelectronics and Lasers at the Physics Department of University of Porto (1998); in 2005 concluded his Ph.D. program at University of Porto with work in thin films of piezoelectric materials for sensor and actuator applications. Since 1995, J.R.A. Fernandes has been involved in several research projects related to the development of functional materials for sensor applications. J.R.A. Fernandes is a senior researcher at INESC TEC since 2005 with work in the biosensing field. Since 200, J.R.A. Fernandes is an assistant professor in the Physics Department of Universidade de Trás-os-Montes e Alto Douro (UTAD), has more than 100 publications in national and international conferences and peer reviewed journals and is also co-author of 1 book chapter.

Paula Martins-Lopes graduated in Agriculture Engineering at the Trás-os-Montes and Alto Douro University (UTAD), Portugal in 1996. She received her M.Sc. degree 
in Genetic Resources and Plant and Forestry Breeding from the UTAD, Portugal in 1999 and her Ph.D. in Genetics from UTAD, Portugal in 2006. The research for the M.Sc. and Ph.D. was performed in collaboration with the Cereals Department of the Cambridge Lab, John Innes Centre, Norwich, U.K. At the present, Paula F. MartinsLopes, is an Assistant Professor at the Department of Genetic and Biotechnology, UTAD, where she lectures $1 \mathrm{st}, 2$ nd and 3 rd cycle studies. She is at present the director of four degrees: 1st cycle in Genetics and Biotechnology, 2nd cycle in Health Science Biotechnology, 2nd Cycle of Comparative Molecular Genetics and Technology and the 3rd cycle of Comparative Molecular Genetics. Paula is also the vice-president of Pedagogical council of the School in Life Science and Environment. Her research lines are linked with studies on food authenticity strategies using PCR and Biosensor platforms, aluminum stress in cereals, pathogen stress in olive and antigenotoxic effects of food using Drosophila melanogaster models. She has been involved in 16 research project and has been the PI of three of them. From her research work, Paula is author and co-author of 32 full-papers published SCI journals, 3 patents, 5 book chapters, 3 abstracts published in SCI journals, 1 full-paper published in peer-reviewed journal, 7 full papers in proceedings and 123 abstracts published in national and international meetings. She has been the supervisor of $5 \mathrm{PhD}$ thesis, 5 master dissertations and more than 20 undergraduate final reports. She has been invited last year to be an evaluator of research reports by the national foundation for science and technology. Paula has referee of several SCI journals. 